\title{
Is there any relationship between initial hematological parameters and severity of scorpion envenomation?
}

\author{
Özden Özgür $\operatorname{Horoz}^{1 \odot}$, Dinçer Yıldızdaş ${ }^{1 \oplus}$, Nagehan Aslan $^{1 \oplus}$, Sinem Sarı Gökay ${ }^{2 \odot}$, \\ Faruk Ekinci $^{1 \oplus}$, Sevcan Erdem ${ }^{3 \odot}$, Zeliha Haytoğlu ${ }^{4 \oplus}$, Yaşar Sertdemir ${ }^{5 \odot}$, \\ Hayri Levent Yilmaz ${ }^{2 \odot}$
}

Divisions of ${ }^{1}$ Pediatric Intensive Care Unit, ${ }^{2}$ Pediatric Emergency and ${ }^{3}$ Pediatric Cardiology, ${ }^{4}$ Department of Pediatric and ${ }^{5}$ Department of Biostatistics, Çukurova University Faculty of Medicine, Adana, Turkey.

\begin{abstract}
Background and objectives. Most cases of severe scorpion envenomation occur in children and are associated with significant morbidity and mortality. Excessive systemic inflammatory response, which leads to multiple organ involvement, is an emerging challenge during severe envenomation. The aim of this study was to investigate if there was any relationship between initial hematological parameters and severe envenomation in pediatric patients presenting with scorpion envenomation.
\end{abstract}

Method. This study was performed retrospectively, at the pediatric emergency unit and pediatric intensive care unit of the Çukurova University Medical School in Turkey. Two hundred and fifty-seven cases with scorpion envenomation, and a control group consisting of one hundred and fifteen healthy children were included in the study.

Results. White blood cell, neutrophil, lymphocyte, platelet, neutrophil/lymphocyte ratio (NLR), platelet/ lymphocyte ratio (PLR) and PDW values of patients were higher than the controls $(\mathrm{p}<0.05)$. Mean NLR was 3.8 \pm 4.7 in patients. Patients were analyzed with the help of the decision tree model, and it was seen that in patients who had applied to hospital in less than an hour after the scorpion sting, $87.5 \%$ of the patients whose NLR value was between the 0.519-1.969 interval (below 2.1 which we found as the cut-off value) did not need to be hospitalized in the intensive care unit, $54.1 \%$ of the patients whose NLR value was higher than 1.969 needed to be hospitalized at the intensive care unit.

Conclusions. Severe envenomation is associated with mortality and morbidity in children. Our findings showed that NLR seems to be a useful tool in predicting severe envenomation.

Key words: children, scorpion, severe envenomation, neutrophil/lymphocyte ratio.

Scorpion envenomation has been a medical problem for all continents (except for Antarctica) for centuries. It is more common in Central and South America, North Africa, the Middle East and South Asia. ${ }^{1}$ There are 1753 types of scorpions known worldwide and 23 types in Turkey. The properties of the scorpion, such as type, age, size, and nutritional status, determine the severity of the envenomation. Also, the

$凶$ Özden Özgür Horoz

oozgurhoroz@yahoo.com

Received 30th July 2019, revised 11th October 2019, accessed 7th November 2019. number of stings, depth of venom injection, location of stings in proximity to the head and neck, and age and health status of the victim determine the severity of envenomation. ${ }^{2}$

Depending on the scorpion, 66 to 90 percent of stings have signs and symptoms limited to local pain, paresthesias, and skin changes without systemic effects. ${ }^{3}$ Systemic effects become apparent in 10 to 33 percent of patients. After envenomation, symptoms may begin immediately and typically progress to maximum severity within 5 hours. The clinical effects of stings are characterized 
by autonomic, cardiovascular, neurological and gastrointestinal effects. All of these are responsible for severe envenomation. Cardiovascular effects are atrial tachycardia, ventricular extrasystoles, T-wave inversion, ST-T wave changes, bundle-branch block. Catecholamine-induced myocarditis and myocardial ischemia results in pulmonary edema and cardiogenic shock. ${ }^{4-6}$ Neurological effects: systemic envenomation is characterized by neuromuscular abnormalities resulting from effects on the somatic and cranial nerves. These include local pain and paresthesias, unexplained agitation or inconsolable crying, dysphagia, drooling, abnormal eye movements with blurred vision, slurred speech, tongue fasciculations, restlessness, fasciculations, shaking and jerking of the extremities, alternating opisthotonos and tetanic forward flexion of the body. ${ }^{7}$ Gastrointestinal effects are vomiting, abdominal pain and diarrhea. Also, acute pancreatitis has been reported..$^{5,8}$

Cardiovascular toxic effects and acute pulmonary edema are the most important complications of scorpion stings and the most frequent cause of death in the first 24 hours after the sting. Most cases of severe envenomation occur in children and are associated with morbidity and mortality. ${ }^{9}$ Although there is no accurate data worldwide, a $20 \%$ mortality rate is reported in untreated infants and $10 \%$ in untreated school-aged children. ${ }^{10}$

Many studies have been conducted recently with the help of hematological parameters, especially, the ratio between the absolute number of neutrophils and the number of lymphocytes has been considered as a potential new biomarker predicting the worse clinical course of cancer, infectious diseases, cardiovascular diseases, end-stage renal disease, immunologic diseases, and schizophrenia. The platelet/lymphocyte ratio is also used to determine inflammation. ${ }^{11-17}$

As it is known, pro-inflammatory and antiinflammatory cytokines and mediators are excreted in scorpion stings. The balance between the pro-inflammatory and anti-inflammatory mediators, in other words, the excessive systemic inflammatory response of the host determines the severity of the envenomation. ${ }^{18}$ Therefore, the aim of this study was to investigate if there was any relationship between initial mean platelet volume (MPV), platelet distribution width (PDW), neutrophil/lymphocyte ratio (NLR) and platelet/lymphocyte ratio (PLR) and severe envenomation in pediatric patients presenting with a scorpion sting.

\section{Material and Methods}

This study was performed retrospectively (between May 3, 2007, and December 31, 2018), at the pediatric emergency unit and pediatric intensive care unit of Çukurova University Medical School in Turkey. Two hundred and fifty-seven cases consisting of patients aged 18 years or younger who were treated with scorpion stings, and a control group consisting of one hundred and fifteen healthy children were included in the study. Children with infectious or inflammatory diseases and chronic disorders were excluded from this study's control group.

The study protocol was approved by the local institutional ethics committee (04.01.2019/84) and was performed in accordance with the Helsinki Declaration.

Age, gender, weight, time and date of presentation, place of first referral, types of scorpions, location, time and number of stings, region of residence (urban or rural), clinical signs and symptoms, administration of antivenom, number of antivenoms, treatment, hospitalization, length of hospital stay, and results were recorded for all patients. The patients were treated with antivenom against the Androctonus Crassicauda produced by Refik Saydam Hygiene Center Presidency.

Hematological parameters, which were obtained easily and economically through complete blood counts, to investigate the relation between the severity of the disease and prognosis were: White blood cells (WBC), 
neutrophil, lymphocyte, platalate count (PLT), MPV, PDW, NLR and PLR were calculated from the complete blood count at admission.

Myocarditis was determined as follows: one finding of cardiac insufficiencies, such as tachycardia, murmur, gallop rhythm, or muffled heart sounds, and at least one of the following: change on ECG, altered cardiac function at Echocardiogram (ECHO), or an increase in troponin levels. ${ }^{19-20}$ ECHO examinations were performed by a pediatric cardiologist.

According to Abroug's previous classification, our patients were categorized based on clinical findings. ${ }^{21}$ According to this, the patients with local signs only were classified as stage I, the patients with local signs extending to the proximal side and/or mild systemic signs were classified as stage II, and the patients with lifethreatening systemic signs were classified as stage III.

\section{Statistical Analysis}

Statistical analysis was performed using IBM SPSS Statistics Version 20.0 statistical software package. Categorical variables were expressed as numbers and percentages, whereas continuous variables were summarized as mean and standard deviation and as median and minimum-maximum where appropriate. For comparison of more than two groups, Oneway ANOVA or Kruskal Wallis test was used depending on whether the statistical hypotheses were fulfilled or not. To evaluate the correlations between measurements, the Pearson Correlation Coefficient or Spearman Rank Correlation Coefficient was used depending on whether the statistical hypotheses were fulfilled or not. A receiver operator characteristic (ROC) curve analysis was performed in order to identify the optimal cut-off point. Decision tree analysis with 10 fold cross-validation was applied to predict the probability of PICU stay. The statistical level of significance for all tests was considered to be 0.05 .22

\section{Results}

We enrolled 257 patients (145 male), and 115 controls (72 male) in the study. There were no differences in age and gender between the patient and control groups. WBC, neutrophil, lymphocyte, platelet, NLR, PLR and PDW values of the patients were higher than the control group's $(p<0.05)$. Demographic and hematologic parameters of patients and control groups are shown in Table I.

According to Abroug's classification, patients were divided into three stages. There was a difference only for NLR value which from stage 1 to 3 , the NLR was increasing and it was statistically significant $(p<0.001)$ but there was no difference for PLR, MPV, and PDW between the stages 1,2 and 3 ( $p>0.05$ ).

There was a statistically significant difference for NLR and PDW values when the patients were separated according to rural or urban areas. The NLR and PLR values of the patients who were admitted to the hospital more than 1 hour after a scorpion sting were significantly higher (respectively $\mathrm{p}<0.001, \mathrm{p}<0.001$ ). Scorpion species were classified as yellow, black and unknown. PDW was higher in unknown species of scorpion stings ( $p<0.001)$. Patients were evaluated in terms of the number of stings and the location of stings but there were no differences for NLR, PLR, MPV, and PDW ( $p>0.05)$. Patients who were stung by scorpions at night had higher PLR values $(p=$ 0.029). Patients who were given antivenom and who had myocarditis had higher NLR values (respectively p <0.001, p <0.001). The NLR and PLR values of the patients who were admitted to the pediatric intensive care unit (PICU) were significantly higher (respectively $\mathrm{p}<0.001, \mathrm{p}=$ $0.020)$, and PDW values were lower $(p=0.004)$. A comparison of epidemiological and clinical characteristics with hematological parameters in patients are shown in Table II. 
Table I. Demographic, and hematologic parameters of patients and controls.

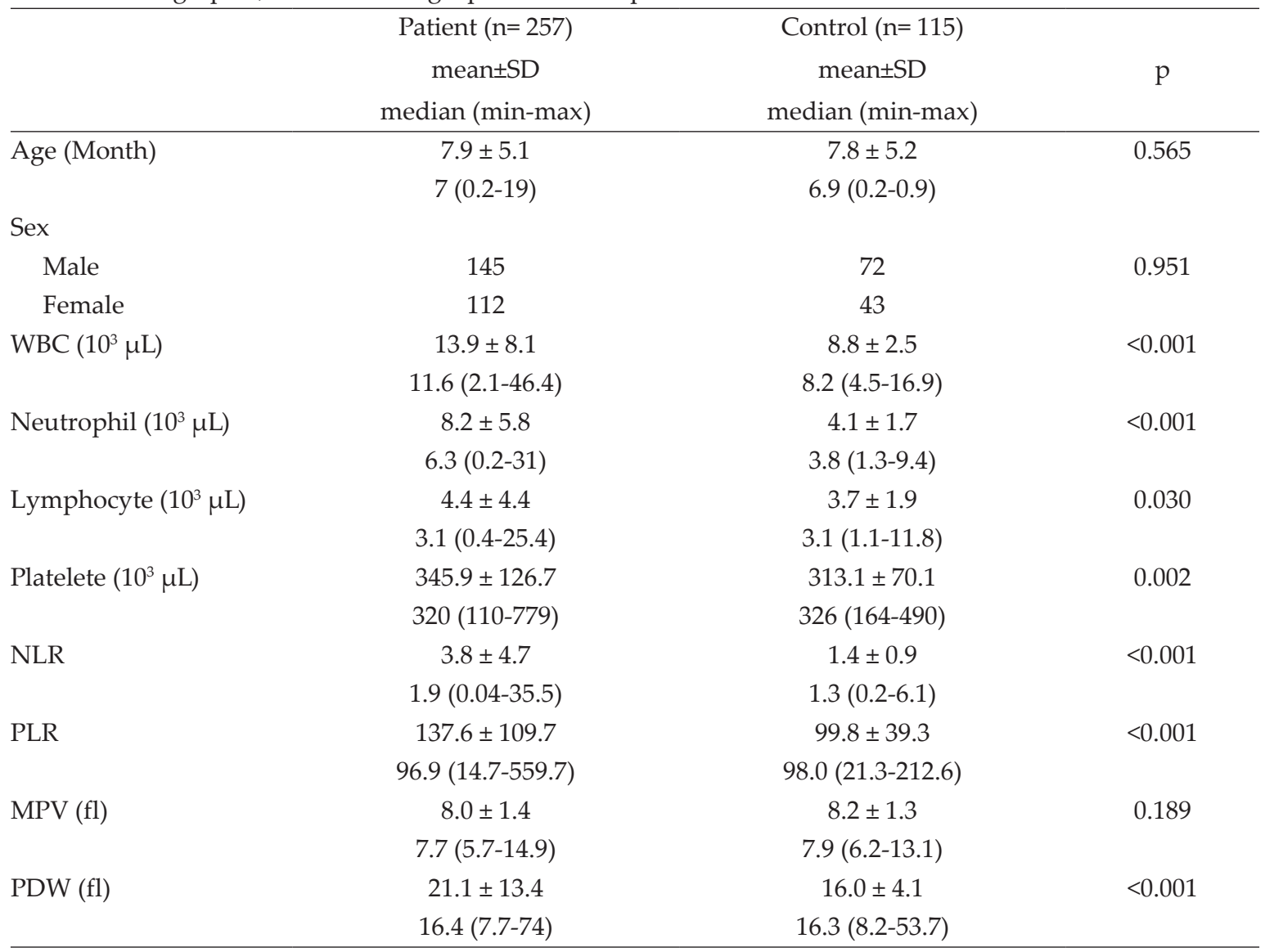

WBC: white blood cell, NLR: neutrophil/lymphocyte ratio lymphocyte, PLR: platelet/lymphocyte ratio, MPV: mean platelet volume, PDW: platelet distribution width.

Mean NLR was $3.8 \pm 4.7$ and was median: 1.9 (minimum: 0.04- maximum: 35.5) in patients $(\mathrm{n}=257)$. One hundred and thirty-five patients $(135 / 250)$ were admitted to the PICU. ROC curve analysis revealed that, in predicting PICU stay, the area under the curve for NLR was 0.643 (95\% CI 0.575-0.711), $\mathrm{p}<0.001$ (Fig. 1). The optimal cut-off point for NLR was obtained as 2.1 with $65.4 \%$ sensitivity and $70.5 \%$ specificity. There was a poor correlation between the length of PICU stay and NLR $(\mathrm{p}<0.001, \mathrm{r}=0.225)$. There was no association between length of PICU stay and PLR, MPV, PDW ( $p>0.05$ for all).

Myocarditis was seen in 36 of the patients (36/135) admitted to the PICU. ROC curve analysis revealed that, in predicting myocarditis, the area under the curve for NLR was $0.671(95 \%$ CI $0.560-0.782$ ), $\mathrm{p}<0.001$ (Fig. 2). The optimal cut-off point for NLR was obtained as 2.5 with $69.4 \%$ sensitivity and $64.2 \%$ specificity.

The decision tree analysis with 10 fold crossvalidation was applied. The decision model generated from the dataset which is shown in Figure 3. All patients were divided into five subgroups (five nodes) from root node (PICU, non PICU) to time of admission (1 hour, more than 1 hour) and finally to NLR $(\leq 0.519,0.519$ $1.969, \geq 1.969$ ). The probability of PICU stays varied from $12.5 \%$ to $75.2 \%$. For example, $71.8 \%$ of the patients who were admitted within the 
Table II. A comparison of epidemiological and clinical characteristics with hematological parameters in patients.

\begin{tabular}{|c|c|c|c|c|}
\hline & $\begin{array}{l}\text { NLR } \\
\text { mean } \pm S D\end{array}$ & $\begin{array}{l}\text { PLR } \\
\text { mean } \pm S D\end{array}$ & $\begin{array}{l}\mathrm{MPV}(\mathrm{fl}) \\
\text { mean } \pm \mathrm{SD}\end{array}$ & $\begin{array}{l}\mathrm{PDW}(\mathrm{fl}) \\
\text { mean } \pm \text { SD }\end{array}$ \\
\hline & median (min-max) & median (min-max) & median (min-max) & median (min-max) \\
\hline District & $4.5 \pm 5.5$ & $144.3 \pm 116.2$ & $8.1 \pm 1.3$ & $15.8 \pm 5.0$ \\
\hline Urban (n=135) & $2.4(0.1-35.5)$ & 100 (14.7-559.7) & $8.0(5.7-11.9)$ & $16.2(7.7-46.6)$ \\
\hline \multirow[t]{2}{*}{ Rural (n=122) } & $2.9 \pm 3.1$ & $130.2 \pm 102.1$ & $7.9 \pm 1.4$ & $25.7 \pm 16.4$ \\
\hline & $1.5(0.04-21.1)$ & 93.7 (26.8-491.5) & $7.5(5.7-14.9)$ & $16.8(8.9-74)$ \\
\hline $\mathrm{p}$ & 0.003 & 0.303 & 0.374 & $<0.001$ \\
\hline Time to admission & $2.5 \pm 3.2$ & $107.8 \pm 83.2$ & $8.1 \pm 1.4$ & $20.4 \pm 13.2$ \\
\hline 1 Hour $(\mathrm{n}=124)$ & $1.3(0.1-21.1)$ & $83.4(27.3-469.6)$ & $7.7(5.7-14.9)$ & $16.3(7.7-74.0)$ \\
\hline \multirow[t]{2}{*}{$>1$ Hour $(n=133)$} & $4.9 \pm 5.4$ & $165.5 \pm 123.7$ & $7.9 \pm 1.4$ & $22.1 \pm 13.7$ \\
\hline & $3.1(0.04-35.5)$ & $134.8(14.7-559.7)$ & $7.7(5.7-11.9)$ & $16.6(9.2-69.0)$ \\
\hline $\mathrm{p}$ & $<0.001$ & $<0.001$ & 0.485 & 0.370 \\
\hline Type of scorpion & $3.5 \pm 3.8$ & $133.1 \pm 105.9$ & $7.9 \pm 1.4$ & $25.2 \pm 16.5$ \\
\hline Unknown (n=127) & $1.9(0.1-26)$ & $93.5(14.7-559.7)$ & $7.5(5.7-14.9)$ & $16.9(7.7-74)$ \\
\hline \multirow[t]{2}{*}{ Black (n=14) } & $3.0 \pm 3.3$ & $107.6 \pm 88.5$ & $8.4 \pm 1.4$ & $15.3 \pm 2.0$ \\
\hline & $1.8(0.4-11.6)$ & $82.2(32.8-368.3)$ & $8.3(5.9-10.9)$ & $16.3(11.1-16.7)$ \\
\hline \multirow[t]{2}{*}{ Yellow (n=116) } & $4.1 \pm 5.4$ & $146.0 \pm 115.8$ & $8.1 \pm 1.3$ & $17.6 \pm 8.5$ \\
\hline & $2.0(0.04-35.5)$ & $103.5(16.7-535.0)$ & $7.9(5.8-12.1)$ & $16.2(9.0-51.1)$ \\
\hline $\mathrm{p}$ & 0.265 & 0.368 & 0.226 & $<0.001$ \\
\hline Time of sting & $3.4 \pm 4.4$ & $126.0 \pm 95.0$ & $8.0 \pm 1.4$ & $20.7 \pm 12.7$ \\
\hline Day $(n=160)$ & $1.8(0.04-35.5)$ & $97.1(14.7-535.0)$ & $7.7(5.7-14.9)$ & $16.5(9.0-69.0)$ \\
\hline \multirow[t]{2}{*}{ Night (n=97) } & $4.3 \pm 4.9$ & $156.8 \pm 128.8$ & $8.0 \pm 1.2$ & $21.9 \pm 14.5$ \\
\hline & $2.7(0.1-26.7)$ & $96.6(16.7-559.7)$ & $8(5.8-10.9)$ & $16.4(7.7-74)$ \\
\hline $\mathrm{p}$ & 0.136 & 0.029 & 0.999 & 0.055 \\
\hline Administration of antivenom & $2.5 \pm 2.7$ & $120.6 \pm 96.6$ & $7.9 \pm 1.4$ & $22.6 \pm 15.1$ \\
\hline No $(n=104)$ & $1.5(0.1-12)$ & $89.6(18.4-491.5)$ & $7.6(5.7-14.9)$ & $16.7(9-74)$ \\
\hline \multirow[t]{2}{*}{ Yes $(n=153)$} & $4.6 \pm 5.4$ & $149.7 \pm 116.7$ & $8.1 \pm 1.3$ & $20.0 \pm 12.0$ \\
\hline & $2.6(0.04-35.5)$ & $113.3(14.7-559.7)$ & $7.9(5.8-12.1)$ & $16.3(7.7-65.4)$ \\
\hline $\mathrm{p}$ & $<0.001$ & 0.056 & 0.662 & 0.359 \\
\hline PICU stay & $2.6 \pm 3.8$ & $119.7 \pm 90.6$ & $8.1 \pm 1.4$ & $19.0 \pm 11.7$ \\
\hline No $(n=122)$ & $1.2(0.3-35.5)$ & $84.5(36.2-535.0)$ & $7.7(5.9-14.9)$ & $16.8(9.0-74.0)$ \\
\hline \multirow[t]{2}{*}{ Yes $(n=135)$} & $4.8 \pm 5.0$ & $155.3 \pm 123.3$ & $7.8 \pm 1.2$ & $24.1 \pm 14.9$ \\
\hline & $2.9(0.04-26.7)$ & $104.8(14.7-559.7)$ & $7.5(5.8-11.9)$ & $16.7(8.9-69.0)$ \\
\hline $\mathrm{p}$ & $<0.001$ & 0.020 & 0.067 & 0.004 \\
\hline Myocarditis & $3.3 \pm 4.2$ & $134.0 \pm 108.3$ & $8.0 \pm 1.4$ & $21.0 \pm 13.3$ \\
\hline No $(n=221)$ & $1.8(0.1-35.5)$ & $93.6(16.7-559.7)$ & $7.7(5.7-14.9)$ & $16.4(7.7-74)$ \\
\hline \multirow[t]{2}{*}{ Yes $(n=36)$} & $6.5 \pm 5.9$ & $159.5 \pm 117.4$ & $8.2 \pm 1.1$ & $21.8 \pm 14.3$ \\
\hline & $5.2(0.04-26)$ & $147.1(14.7-486.0)$ & $8.3(6.3-10.2)$ & $16.3(10.2-65.4)$ \\
\hline $\mathrm{p}$ & $<0.001$ & 0.197 & 0.395 & 0.767 \\
\hline
\end{tabular}

NLR: neutrophil/lymphocyte ratio lymphocyte, PLR: platelet/lymphocyte ratio, MPV: mean platelet volume, PDW: platelet distribution width, PICU: pediatric intensive care unit. 


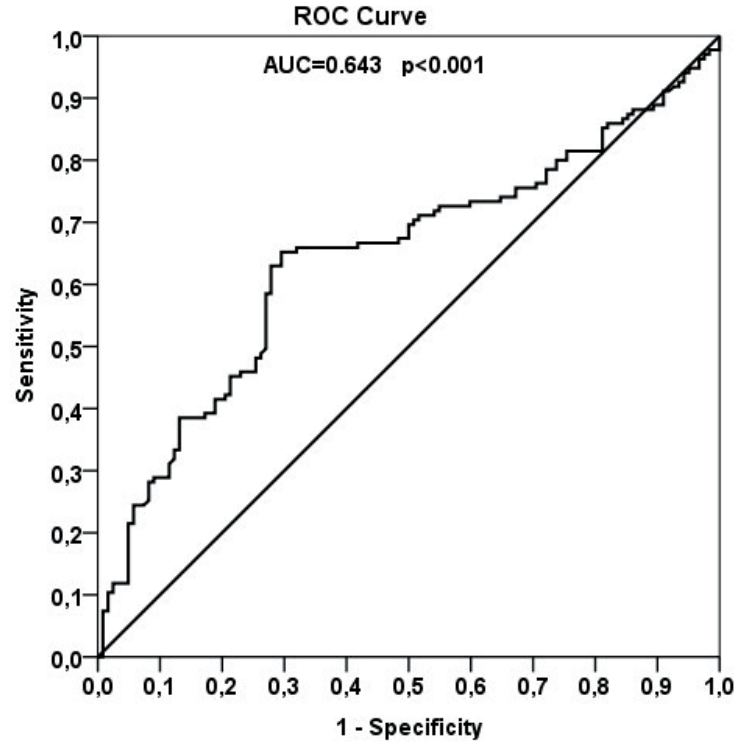

Fig. 1. ROC curve analysis of NLR predicts pediatric intensive care unit stay. Area under curve (AUC): 0.643 .

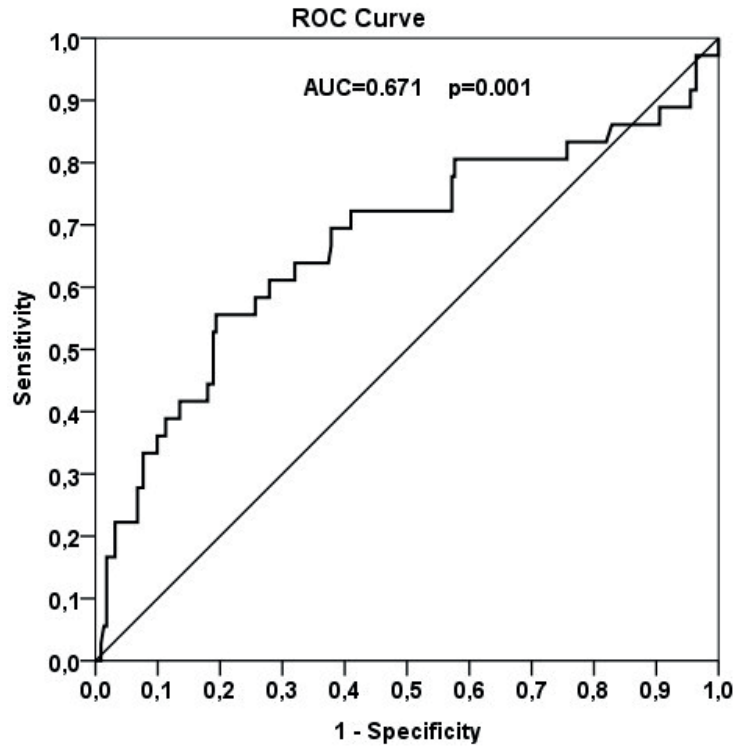

Fig. 2. ROC curve analysis of NLR predicts myocarditis. Area under curve (AUC): 0.671.

\section{PICU}

\begin{tabular}{|l|c|c|}
\hline \multicolumn{4}{|c|}{ Node 0 } \\
Categony & $\psi$ & n \\
\hline NON-PICU & 47.5 & 122 \\
N PICU & 52.5 & 135 \\
\hline Total & 100.0 & 257 \\
\hline
\end{tabular}

Time to admission

Adj. P-walue $=0,000$. Chi-square $=56$. 756. $\mathrm{df}=1$

\begin{tabular}{|l|r|r|r|}
\hline PICU & 15 & 120 & $88,9 \%$ \\
\hline $\begin{array}{l}\text { Overall } \\
\text { Percentage }\end{array}$ & $33,9 \%$ & $66,1 \%$ & $74,7 \%$ \\
\hline
\end{tabular}
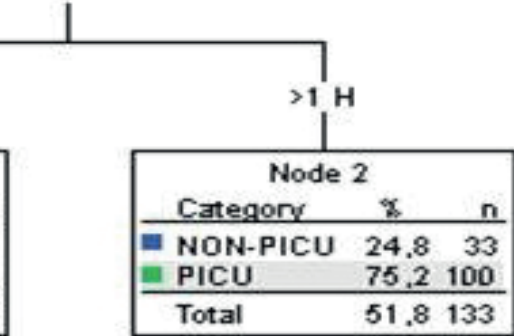

adj. P-value $=0,001$. Chi-square $=21$. 999, $\mathrm{df}=2$

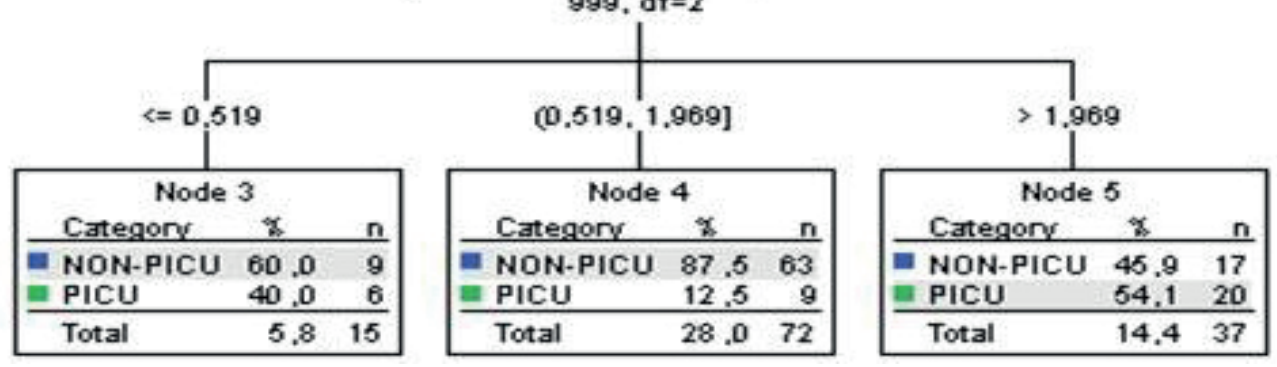

Fig. 3. The decision tree model. 
first hour of stinging did not require intensive care. Whereas, $75.2 \%$ of the patients who were admitted after more than one hour required intensive care stay. Additionally, $87.5 \%$ of the patients who applied within the first hour and whose NLR values were in the range of 0.5191.969 did not require intensive care stay. (88.9\% sensitivity, $59 \%$ specificity, positive predictive value $70.59 \%$, negative predictive value $82.76 \%$, accuracy $74.71 \%$ )

\section{Discussion}

Pro-inflammatory and anti-inflammatory cytokines and mediators are excreted in scorpion stings. The balance between the proinflammatory and anti-inflammatory mediators, in other words, the excessive systemic inflammatory response of the host determines the severity of the envenomation. ${ }^{18}$ In addition, the properties of the scorpion, number of stings, depth of venom injection, location of stings, age and health status of the victim determine the severity of the envenomation. Severe envenomation is associated with morbidity and mortality. Therefore, it is the reason for hospitalization and increased costs. When the literature was reviewed, no study was found which investigated the relation between initial hematological parameters and the disease severity in scorpion envenomations. The correlation between platelet indices and scorpion envenomation was assessed only in one study. ${ }^{23}$ To the best of our knowledge, our study is the first to investigate the role of initial hematological parameters in the severity of scorpion envenomation in children. When we compared our patients with healthy controls, WBC, neutrophil, lymphocyte, platelet count, PDW, NLR and PLR ratios were statistically significantly higher, whereas the MPV values of our patients were lower than the controls. However, this situation was not statistically significant.

In previous studies, leucocytosis has been reported in patients who were admitted due to scorpion stings. $18,24,25$ In our study, it was determined that the WBC values of the patients were significantly higher than the control groups'.

Various studies have been published in which the MPV value in inflammatory diseases have been investigated. It has been reported in these studies (chronic urticaria, rheumatoid arthritis, chronic hepatitis B, myocardial infarction, an acute attack of FMF) that the MPV value is high in association with the severity of inflammation. ${ }^{26-30}$ However, it has also been determined that MPV is in lower values in cystic fibrosis acute exacerbation. ${ }^{31}$ In our study, the MPV values of our patients were found to be lower however, this was not statistically significant.

PDW value, which is one of the hematological parameters, has been stated to be associated with inflammation in many diseases: acute coronary syndrome, heart failure, sickle-cell disease, tuberculosis, bacteremia, chronic urticaria. ${ }^{26,32-34}$ In our study, the PDW values of our patients who were admitted due to scorpion stinging, were found to be statistically significantly higher than the control group.

As an indicator of the inflammatory period, PLR has been the subject of many studies recently. The association between the inflammatory period indicators and disease have been investigated even in psychiatric diseases..$^{30,35-37}$ The PLR value was statistically significantly higher in our patients as well.

NLR which is prominent due to rapidity, easy detection and cost-effectiveness is being reported with increasing frequency as a reliable biomarker of systemic inflammation. ${ }^{38-40}$ Djordjevic et al. ${ }^{16}$ have investigated the relationship between hematological parameters and prognosis in critically ill patients with bacteremia, and have reported that high MPV and high NLR are very good independent predictors in predicting lethal outcome. It has been reported that NLR is a useful tool for clinical severity and prognosis in inflammatory and malignant diseases. ${ }^{41-43}$ The mean NLR in our patients was $3.8 \pm 4.7$ and statistically 
significantly higher than the control group. This suggested that it was related to severe inflammation post scorpion stinging.

In patients who applied from urban areas the NLR was found to be higher, whereas in scorpion stinging which happened at night the PLR values were found to be higher, and in patients who applied to the hospital later than an hour post scorpion stinging both the NLR and PLR values were significantly higher and this suggested increasingly inflammation response. When considered according to scorpion types there was no difference between the scorpion types for NLR, PLR, and MPV, however, the PDW value was higher in stings with unknown scorpion types. When the patients were divided according to Abroug's calcification, NLR significantly increased from stage 1 to stage 3 and this supported the idea that when envenomation severity increased the NLR increased. Additionally, the fact that the NLR value was significantly higher in patients who were given antivenoms, patients who needed intensive care and patients who had myocarditis was considered as related to the severity of the disease.

In the prediction of admission of patients to intensive care due to scorpion stings, initial NLR was a useful parameter. The optimal cut-off point for NLR was obtained as 2.1 with $65.4 \%$ sensitivity and $70.5 \%$ specificity. When the literature is reviewed, various publications about NLR cut-off value in children can be found. The NLR cut-off value has been reported as 1.97 in predicting sepsis, as 2.86 in gastrointestinal hemorrhage in Henoch-Schönlein purpura, like 1.4 to assess the inflammation in rheumatoid arthritis and as 2.51 in Kawasaki disease. ${ }^{15,44-46}$ However, in the literature, there is no cut-off value for the prediction of severity in children who applied with scorpion stings. In a retrospective study that evaluated adult patients who applied with snake bites, it was reported that the patients with a high NLR value were found to have a longer hospital stay. ${ }^{47}$ Nevertheless, we have determined a weak correlation between the intensive care unit stay duration and NLR. We have determined that NLR can be used in predicting myocarditis which is another picture that indicates the severity of scorpion envenomation. The optimal cut-off point for NLR was obtained as 2.5 with $69.4 \%$ sensitivity and $64.2 \%$ specificity for myocarditis.

When 257 patients, who were admitted to the hospital due to scorpion stings, were analyzed with the help of the decision tree model it was seen that $71.8 \%$ of the patients who were admitted to the emergency department within the first hour did not need to be hospitalized at the intensive care unit. On the other hand, $75.2 \%$ of the patients who were admitted to the hospital later than an hour after the scorpion sting needed to be hospitalized in the intensive care unit. In patients who had applied to the hospital in less than an hour after the scorpion sting $87.5 \%$ of the patients whose NLR value was between the 0.519-1.969 interval (below 2.1 which we found as the cut-off value) did not need to be hospitalized in the intensive care unit, $54.1 \%$ of the patients whose NLR value was higher than 1.969 needed to be hospitalized in the intensive care unit. Interestingly, six $(40 \%)$ out of the 15 patients whose NLR value was lower than 0.519 and who had applied to the hospital within the first hour after the scorpion sting also needed to be hospitalized in the intensive care unit. When these six children whose NLR values were low were examined, it was found that they were all under 1 year old and their lymphocyte and neutrophil counts were (7.7-25.3) and (2.6-8.0) respectively. As it is known, there is lymphocyte dominance in children under 1-year-old and this age group in under risk for severe envenomation after scorpion stings. The fact that these six children had lymphocytosis, and were younger than one-year-old explains why they needed to be hospitalized at in intensive care units despite having low NLR values.

The major limitations of our study were being retrospective, being the only center and including pediatric patients with restricted racial properties. 
In conclusion, systemic inflammation occurs after scorpion stings and the degree of inflammation determines severe envenomation. After scorpion stings, severe envenomation risk increases especially in pediatric patients. ${ }^{48}$ This situation is associated with mortality and morbidity. High NLR, which will be calculated from the complete blood count at the admission of the patient to the hospital, can predict severe envenomation. High NLR in scorpion stings may be helpful during the decision stage. High NLR in scorpion stings may be a helpful tool in deciding when the patient should be transported to a more advanced center than the sub-urban areas where there is limited access to severe envenomation treatment and especially patient care resources. However, we believe that more studies are needed about NLR, which is quite popular lately, and other hematological parameters.

\section{REFERENCES}

1. Skolnik AB, Ewald MB. Pediatric scorpion envenomation in the United States: morbidity, mortality, and therapeutic innovations. Pediatr Emerg Care 2013; 29: 98-103.

2. Gökay SS, Kendir ÖT, Güllü UU, et al. Myocarditis and early markers of cardiac response associated with scorpion stings in children. Wilderness Environ Med 2018; 29: 471-478.

3. Cupo P. Clinical update on scorpion envenoming. Rev Soc Bras Med Trop 2015; 48: 642-649.

4. Bahloul M, Chaari A, Dammak H, et al. Pulmonary edema following scorpion envenomation: mechanisms, clinical manifestations, diagnosis and treatment. Int J Cardiol 2013; 162: 86-91.

5. Poon-King T. Myocarditis from scorpion stings. Br Med J 1963; 1: 374-377.

6. Bahloul M, Ben Hamida C, Chtourou K, et al. Evidence of myocardial ischaemia in severe scorpion envenomation. Myocardial perfusion scintigraphy study. Intensive Care Med 2004; 30: 461-467.

7. Clark RF, Selden BS, Kunkel DB, Frost MD. Abnormal eye movements encountered following severe envenomations by Centruroides sculpturatus. Neurology 1991; 41: 604.
8. Otero R, Navío E, Céspedes FA, et al. Scorpion envenoming in two regions of Colombia: clinical, epidemiological and therapeutic aspects. Trans $\mathrm{R}$ Soc Trop Med Hyg 2004; 98: 742-750.

9. Gökay SS, Yilmaz HL, Yildizdas RD, et al. A relationship between clinical and laboratory characteristics in children with severe scorpion envenomation in Çukurova, Turkey. Pediatr Emerg Care 2018; doi: 10.1097/PEC.0000000000001483. Online ahead of print.

10. Cheng D. Scorpion Envenomation. Medscape. https://emedicine.medscape.com/article/168230overview. (2018, Nov 09) Retrieved 5/19/2019 from https://emedicine.medscape.com/article/168230overview\#a7.

11. Ozdin S, Boke O. Neutrophil/lymphocyte, platelet/ lymphocyte and monocyte/lymphocyte ratios in different stages of schizophrenia. Psychiatry Res 2019; 271: 131-135.

12. Diem S, Schmid S, Krapf M, et al. Neutrophilto-Lymphocyte ratio (NLR) and Platelet-toLymphocyte ratio (PLR) as prognostic markers in patients with non-small cell lung cancer (NSCLC) treated with nivolumab. Lung Cancer 2017; 111: 176181.

13. Dolan RD, Laird BJA, Horgan PG, McMillan DC. The prognostic value of the systemic inflammatory response in randomised clinical trials in cancer: a systematic review. Crit Rev Oncol Hematol 2018; 132: 130-137.

14. Park JS, Seo KW, Choi BJ, et al. Importance of prognostic value of neutrophil to lymphocyte ratio in patients with ST-elevation myocardial infarction. Medicine (Baltimore) 2018; 97: e13471.

15. Chandrashekara S, Mukhtar Ahmad M, Renuka $\mathrm{P}$, Anupama KR, Renuka K. Characterization of neutrophil-to-lymphocyte ratio as a measure of inflammation in rheumatoid arthritis. Int J Rheum Dis 2017; 20: 1457-1467.

16. Djordjevic D, Rondovic G, Surbatovic M, et al. Neutrophil-to-lymphocyte ratio, monocyte-tolymphocyte ratio, platelet-to-lymphocyte ratio, and mean platelet volume-to-platelet count ratio as biomarkers in critically ill and injured patients: which ratio to choose to predict outcome and nature of bacteremia? Mediators Inflamm 2018; 2018: 3758068.

17. Turkmen K, Erdur FM, Ozcicek F, et al. Platelet-tolymphocyte ratio better predicts inflammation than neutrophil-to-lymphocyte ratio in end-stage renal disease patients. Hemodial Int 2013; 17: 391-396.

18. Petricevich VL. Scorpion venom and the inflammatory response. Mediators Inflamm 2010; 2010: 903295. 
19. Canter CE, Simpson KP. Diagnosis and treatment of myocarditis in children in the current era. Circulation 2014; 129: 115-128.

20. Caglar A, Kose H, Babayigit A, Oner T, Duman M. Predictive factors for determining the clinical severity of pediatric scorpion envenomation cases in southeastern Turkey. Wilderness Environ Med 2015; 26: 451-458.

21. Abroug F, Nouira S, Saguiga H. Envenomations scorpionniques: avences chimiques, physiopathologiques et therapeutiquis. Monograph 1994: 1-68.

22. IBM Corp. Released 2011. IBM SPSS Statistics for Windows, Version 20.0. Armonk, NY: IBM Corp.

23. Konca C, Tekin M, Colak P, Uckardes F, Turgut M. An overview of platelet indices for evaluating platelet function in children with scorpion envenomation. EXCLI J 2014 25; 13: 801-808

24. Borges CM, Silveira MR, Aparecida M, Beker CL, Freire-Maia L, Teixeira MM. Scorpion venominduced neutrophilia is inhibited by a PAF receptor antagonist in the rat. J Leukoc Biol 2000; 67: 515-519.

25. Amucheazi AO, Umeh BU. Scorpion sting pain: which way to treat? Niger J Clin Pract 2012; 15: 9394.

26. Chandrashekar L, Rajappa M, Sundar I, et al. Platelet activation in chronic urticaria and its correlation with disease severity. Platelets 2014; 25: 162-165.

27. Yazici S, Yazici M, Erer B, et al. The platelet indices in patients with rheumatoid arthritis: mean platelet volume reflects disease activity. Platelets 2010; 21: 122-125.

28. Turhan O, Coban E, Inan D, Yalcin AN. Increased mean platelet volume in chronic hepatitis B patients with inactive disease. Med Sci Monit 2010; 16: CR202-CR205.

29. Balcik ÖS, Bilen S, Ulusoy EK, et al. Thrombopoietin and mean platelet volume in patients with ischemic stroke. Clin Appl Thromb Hemost 2013; 19: 92-95.

30. Yorulmaz A, Akbulut H, Taş SA, Tiraş M, Yahya İ, Peru H. Evaluation of hematological parameters in children with FMF. Clin Rheumatol 2019; 38: 701707.

31. Uysal P, Tuncel T, Olmez D, Babayigit A, Karaman $\mathrm{O}$, Uzuner $\mathrm{N}$. The role of mean platelet volume predicting acute exacerbations of cystic fibrosis in children. Ann Thorac Med 2011; 6: 227-230.

32. Vatankulu MA, Sonmez O, Ertas G, et al. A new parameter predicting chronic total occlusion of coronary arteries: platelet distribution width. Angiology 2014; 65: 60-64.
33. Amin MA, Amin AP, Kulkarni HR. Platelet distribution width (PDW) is increased in vasoocclusive crisis in sickle cell disease. Ann Hematol 2004; 83: 331-335.

34. Patrick CH, Lazarchick J. The effect of bacteremia on automated platelet measurements in neonates. Am J Clin Pathol 1990; 93: 391-394.

35. Özyurt G, Binici NC. The neutrophil-lymphocyte ratio and platelet-lymphocyte ratio in adolescent obsessive-compulsive disorder: does comorbid anxiety disorder affect inflammatory response? Psychiatry Res 2019; 272: 311-315.

36. Li Y, Wang B, Zhou S, et al. Do routine blood test results help in the diagnosis of spine tumors? A retrospective study of the significance of pretreatment neutrophil-to-lymphocyte and platelet-to-lymphocyte ratios from 503 spine tumor patients. Medicine (Baltimore) 2019; 98: e14902.

37. Rungsakulkij N, Mingphruedhi S, Suragul W, Tangtawee P, Muangkaew P, Aeesoa S. Plateletto-lymphocyte ratio and large tumor size predict microvascular invasion after resection for hepatocellular carcinoma. Asian Pac J Cancer Prev 2018; 19: 3435-3441.

38. Pascual-González Y, López-Sánchez M, Dorca J, Santos S. Defining the role of neutrophil-tolymphocyte ratio in COPD: a systematic literature review. Int J Chron Obstruct Pulmon Dis 2018; 13: 3651-3662.

39. Kurtipek E, Bekci TT, Kesli R, Sami SS, Terzi Y. The role of neutrophil-lymphocyte ratio and plateletlymphocyte ratio in exacerbation of chronic obstructive pulmonary disease. J Pak Med Assoc 2015; 65: 1283-1287.

40. Taylan M, Demir M, Kaya H, et al. Alterations of the neutrophil-lymphocyte ratio during the period of stable and acute exacerbation of chronic obstructive pulmonary disease patients. Clin Respir J 2017; 11: 311-317.

41. Cedrés S, Torrejon D, Martínez A, et al. Neutrophil to lymphocyte ratio (NLR) as an indicator of poor prognosis in stage IV non-small cell lung cancer. Clin Transl Oncol 2012; 14: 864-869.

42. Donaldson GC, Seemungal TA, Patel IS, et al. Airway and systemic inflammation and decline in lung function in patients with COPD. Chest 2005; 128: 1995-2004.

43. Yu Y, Qian L, Cui J. Value of neutrophil-tolymphocyte ratio for predicting lung cancer prognosis: a meta-analysis of 7,219 patients. Mol Clin Oncol 2017; 7: 498-506. 
44. Dursun A, Ozsoylu S, Akyildiz BN. Neutrophil-tolymphocyte ratio and mean platelet volume can be useful markers to predict sepsis in children. Pak J Med Sci 2018; 34: 918-922.

45. Hong SH, Kim CJ, Yang EM. Neutrophil-tolymphocyte ratio to predict gastrointestinal bleeding in Henoch: Schönlein purpura. Pediatr Int 2018; 60: 791-795.

46. Chen Y, Hua Y, Zhang C, et al. Neutrophilto-lymphocyte ratio predicts intravenous immunoglobulin-resistance in infants under 12-months old with Kawasaki disease. Front Pediatr 2019; 7: 81.
47. Elbey B, Baykal B, Yazgan UC, Zengin Y. The prognostic value of the neutrophil/lymphocyte ratio in patients with snake bites for clinical outcomes and complications. Saudi J Biol Sci 2017; 24: 362-366.

48. Zengin N, Anıl M, Anıl AB, et al. Ege Bölgesinde çocuklarda akrep sokmasının klinik özellikleri: bir Eğitim ve Araştırma Hastanesi deneyimi. Çocuk Acil ve Yoğun Bakım Dergisi 2016; 3: 69-75. 